\title{
SEMS and Sensibility: Self-Expandable Metal Stents for Malignant Biliary Obstruction-Are Stent Characteristics Important?
}

\author{
Evangelos Kalaitzakis ${ }^{1,2,3,4}$
}

Published online: 30 July 2019

(c) Springer Science+Business Media, LLC, part of Springer Nature 2019

Self-expandable metal stents (SEMSs) are an essential component of endoscopic treatment of malignant biliary strictures. Several studies have compared SEMS with plastic stents for palliative therapy of patients with malignant obstruction of the bile duct [1], particularly patients with an expected survival longer than 3 months, and preoperatively [2], including, but not limited to, those receiving neoadjuvant therapy. SEMS are consistently associated with longer stent patency and fewer re-interventions compared with plastic stents. In the preoperative setting, the two stent types do not differ significantly in terms of surgical morbidity or mortality [2]. The use of SEMS in distal malignant biliary obstruction is recommended by all major endoscopy associations, including the European Society of Gastrointestinal Endoscopy [3].

Covered versus uncovered SEMSs in this setting have similar efficacy in terms of stent dysfunction and overall complications [3]. Nevertheless, comparative published data are mixed, with reports supporting an association between covered SEMS and a lower risk of tumor ingrowth, and thus longer stent patency, as well as a higher risk of stent migration, tumor overgrowth, sludge formation, and cholecystitis (due to obstruction of the cystic duct orifice) [4]. Although this issue has been explored in several studies, a clear distinction between fully covered and partially covered SEMSs was not always apparent according to a recent meta-analysis [4]. Furthermore, comparative data on the efficacy of 8-mm

Evangelos Kalaitzakis

kalvag@hotmail.com

1 Digestive Disease Center, Copenhagen University Hospital, Herlev, Denmark

2 Medical School, University of Copenhagen, Copenhagen, Denmark

3 Department of Gastroenterology, University Hospital of Heraklion, Heraklion, Greece

4 Division of Internal Medicine, Medical School, University of Crete, Heraklion, Crete, Greece versus 10-mm SEMS for malignant biliary strictures are scarce.

In this issue of Digestive Diseases and Sciences, Shamah et al. [5] present data from their tertiary care center with regard to the utility of partially covered SEMS compared with uncovered SEMS in patients with malignant biliary strictures. In their series, which is one of the largest in the literature thus far, 213 patients received partially covered SEMS and 65 uncovered SEMS. The two groups did not differ significantly regarding clinical success $(98.1 \%$ vs. $95.5 \%$ ), stent patency duration (302.5 vs. 225.5 days), or overall adverse events (15.5\% vs. $18.5 \%)$. In particular, the rates of stent migration and cholecystitis were similar between the two groups. Importantly, stent diameter $(8 \mathrm{~mm}$ vs. $10 \mathrm{~mm}$ ) did not significantly impact clinical success, stent patency, or overall adverse events.

As the authors acknowledge, there are certain shortcomings (mainly related to its retrospective nature) that should be taken into consideration when interpreting the results of their study. First, the choice of stent type and diameter was at the discretion of the endoscopist. Thus, although the groups receiving partially covered and uncovered stents were comparable, selection bias may have been present. Notably, information regarding prior cholecystectomy or, more importantly, tumor involvement of the cystic duct orifice was not collected, which may have affected the estimated occurrence of cholecystitis [6]. Also, disease stage and the potential effect of oncologic therapy (i.e., chemoradiation) were not accounted for. The same is true for deaths occurring during the study period, which, as competing risks, may have affected estimations of cumulative incidence of stent dysfunction [7]. Despite these issues, the study of Shamah et al. [4] is one of the largest to date comparing partially covered SEMS and uncovered SEMS that in turn contributes to an accumulating amount of evidence indicating that the two stent types do not differ significantly in terms of stent dysfunction. Although their study could not reproduce recent meta-analysis data suggesting longer patency duration 
for covered SEMS in general [4], it is conceivable that the uncoated ends of former may have equalized migration rates between the two groups. Given the mixed results of prior studies regarding the comparative efficacy of partially covered versus uncovered SEMS and the limitations of the study of Shamah et al. [5], further clinical trials are warranted.

The authors also addressed the neglected issue of SEMS diameter in the treatment of malignant biliary obstruction. There have been concerns that SEMS with a larger diameter, and thus with higher axial force, may induce cholecystitis [6] and ischemic changes in tight strictures which, in turn, may propagate tissue hyperplasia and ingrowth with resultant stent occlusion. The presented data do not argue for any important impact of SEMS diameter on stent patency or overall adverse event rate. The occurrence of cholecystitis was also similar between the two groups. These findings lend support to the initial use of either SEMS size $(8 \mathrm{~mm}$ or $10 \mathrm{~mm}$ ) and are in line with data from a recent prospective study that found that 8-mm fully covered SEMSs were noninferior to $10-\mathrm{mm}$ fully covered SEMSs in terms of time to recurrent biliary obstruction [8]. Nonetheless, recently published data have shown that the success rate of endoscopic revisionary stent insertion for SEMS dysfunction in patients with malignant hilar obstruction was higher in the $10-\mathrm{mm}$ stent group than in the $8-\mathrm{mm}$ one $(68 \%$ vs. $31 \%, p=0.044)$. Thus, 10-mm SEMSs may be more suitable for endoscopic re-intervention, which is not uncommon in these patients [9].

Recent advances in stent technology may soon shift the debate from SEMS coating and diameter to different stent characteristics or stent types altogether. Concerns over compromised stent patency following SEMS insertion have led to the development of drug-eluting stents. Although in a recent randomized trial paclitaxel-eluting stents reduced tumor size, they were not superior to conventional covered SEMS regarding time to recurrent biliary obstruction [10]. Despite this, the use of drug-eluting stents in patients with malignant biliary obstruction is intriguing with more research awaited. Furthermore, in a recent multicenter randomized trial, covered SEMS with an anti-migration system had longer patency duration than uncovered SEMS [11]. As duodeno-biliary reflux is related to sludge formation and stent occlusion, SEMS with anti-reflux mechanisms tested in a small randomized trial had longer patency duration compared with conventional SEMS [12]. Finally, 3D printing of bio-synthetic biliary stents, such as plastic stents impregnated with barium along with a stem cell-collagen-cholangiocyte coating, may enable the development of personalized bio-integrating stents that could, at least in theory, resolve several issues characteristic of conventional stents [13].

In summary, partially covered SEMS appears to have similar patency duration, stent dysfunction rates, and adverse event rates compared to uncovered SEMS in patients with malignant biliary obstruction. Although SEMS diameter
( $8 \mathrm{~mm}$ vs. $10 \mathrm{~mm}$ ) does not appear to have any significant impact on stent clinical efficacy, there is an argument that 10-mm SEMS may be more suitable for re-intervention in the event of stent dysfunction [9]. In view of the shortcomings of the current retrospective study and the mixed results of previous studies evaluating partially covered versus uncovered stents in patients with malignant biliary obstruction, larger prospective trials are warranted to evaluate the potential role of SEMS coating in these patients. Advancements in stent technology may, in the near future, allow for new stent designs such as stents with anti-migratory or antireflux mechanisms, drug-eluting stents, or personalized biointegrating stents being adopted.

\section{Compliance with Ethical Standards}

Conflicts of interest The author declares that they have no conflict of interest.

\section{References}

1. Almadi T, Barkun A, Martel M. Plastic vs. self-expandable metal stents for palliation in malignant biliary obstruction: a series of meta-analyses. Am J Gastroenterol. 2017;112:260-273.

2. Crippa S, Cirocchi R, Partelli S, et al. Systematic review and meta-analysis of metal versus plastic stents for preoperative biliary drainage in resectable periampullary or pancreatic head tumors. Eur J Surg Oncol. 2016;42:1278-1285.

3. Dumonceau JM, Tringali A, Papanikolaou IS, et al. Endoscopic biliary stenting: indications, choice of stents, and results: European Society of Gastrointestinal Endoscopy (ESGE) Clinical Guideline-Updated October 2017. Endoscopy. 2018;50:910-930.

4. Saleem A, Leggett CL, Murad MH, et al. Meta-analysis of randomized trials comparing patency of covered and uncovered selfexpandable metal stents for palliation of distal malignant bile duct obstruction. Gastrointest Endosc. 2011;74:321-327.

5. Shamah SP, Chapman CG, Haider H, et al. Partially covered vs uncovered self-expandable metal stents: coating nor diameter affect clinical outcomes. Dig Dis Sci. (Epub ahead of print). https ://doi.org/10.1007/s10620-019-05643-1.

6. Nakai Y, Isayama H, Kawakubo K, et al. Metallic stent with high axial force as a risk factor for cholecystitis in distal malignant biliary obstruction. J Gastroenterol Hepatol. 2014;29:1557-1562.

7. Hamada T, Nakai Y, Isayama H, et al. Estimation and comparison of cumulative incidences of biliary self-expandable metallic stent dysfunction accounting for competing risks. Dig Endosc. 2014;26:270-275.

8. Kawashima H, Hashimoto S, Ohno E, et al. Comparison of 8and $10 \mathrm{~mm}$ diameter fully covered self-expandable metal stents: a multicenter prospective study in patients with distal malignant biliary obstruction. Dig Endosc. 2019;31:439-447.

9. Naitoh I, Nakazawa T, Ban T, et al. 8-mm vs. 10-mm diameter self-expandable metallic stent in bilateral endoscopic stent-instent deployment for malignant hilar biliary obstruction. J hepatobiliary Pancreat Sci. 2015;22:396-401. 
10. Jang SI, Lee KT, Choi JS, et al. Efficacy of a placlitaxel-eluting biliary metal stent with sodium caprate in malignant biliary obstruction: a prospective randomized comparative study. Endoscopy. 2018. https://doi.org/10.1055/a-0754-5763.

11. Kitano M, Yamashita Y, Tanaka K, et al. Covered self-expandable metal stents with an anti-migration system improve patency duration without increased complications compared with uncovered stents for distal biliary obstruction caused by pancreatic carcinoma: a randomized multicenter trial. Am J Gastroenterol. 2013; 108:1713-1722.

12. Lee YN, Moon JH, Choi HJ, et al. Effectiveness of a newly designed antireflux valve metal stent to reduce duodenobiliary reflux in patients with unresectable distal malignant biliary obstruction: a randomized, controlled pilot study. Gastrointest Endosc. 2016;83:404-412.

13. Boyer CJ, Boktor M, Samant H, et al. 3D printing for bio-synthetic biliary stents. Bioengineering. 2019;6:16.

Publisher's Note Springer Nature remains neutral with regard to jurisdictional claims in published maps and institutional affiliations. 